\section{Rheumatoide Arthritis: Wie gut funktioniert die Treat-to-Target-Strategie?}

Ramiro $S$ et al. Is treat-to-target really working in rheumatoid arthritis? a longitudinal analysis of a cohort of patients treated in daily practice (RA BIODAM). Ann Rheum Dis 2020; 79: 453-459. doi:10.1136/annrheumdis-2019-216819

Randomisierte Untersuchungen belegen den Erfolg einer sogenannten Treat-to-Target-Strategie bei der rheumatoiden Arthritis (RA). Ob auch nicht selektierte Patienten unter alltäglichen Praxisbedingungen von diesem Vorgehen profitieren, ist allerdings noch unklar. Ein Team internationaler Wissenschaftler ging dieser Frage im Rahmen einer longitudinalen Kohortenstudie, der RA BIODAM-Studie, nach.

An der internationalen prospektiven Beobachtungsstudie nahmen 571 Patienten aus 10 Ländern teil, welche an einer aktiven RA (44-joint disease activity score/DAS44 $>2,4$ ) litten und neu auf konventionelle oder biologische DMARDs (disease-modifying anti-rheumatic drugs) eingestellt wurden bzw. einen Therapiewechsel vollzogen. Mit Biologika vorbehandelte Patienten schlossen die Forscher von der Studienteil- nahme aus. Alle Patienten wurden über einen Zeitraum von 2 Jahren in vierteljährlichen Intervallen bezüglich der Krankheitsaktivität beurteilt und entsprechend in Form einer Treat-to-Target-Strategie behandelt. Das Therapieziel bildete dabei die Remission (DAS44 < 1,6): Wurde sie nicht erreicht, erfolgte die Therapieintensivierung durch Dosissteigerung oder additiver Wirkstoffgabe (biologische DMARDs, konventionelle DMARDs, Kortikosteroide). Bei Eintritt der Remission wurde dagegen die Therapieintensität nicht verändert. Als primären Studienendpunkt definierten die Wissenschaftler das Erreichen der DAS44Remission zum nächsten 3-Monats-Kontrolltermin. Ferner objektivierten sie die Remissionsrate gemäß 28-joint disease activity score-erythrocyte sedimentation rate (DAS28-ESR), Clinical Disease Activity Index (CDAI), Simplified Disease Activity Index (SDAI) sowie American College of Rheumatology/European League Against Rheumatism (ACR/EULAR)-Definition.

\section{Ergebnisse}

Die Studienteilnehmer (78\% Frauen) waren im Schnitt 56 Jahre alt und litten durchschnittlich seit 6,5 Jahren an der RA. Insgesamt werteten die Forscher 4356 Behandlungstermine aus. Bei $59 \%$ dieser Termine wurde die Treat-to-Target-Strategie korrekt angewendet, in den übrigen 3 bzw. 38 \% dagegen nicht, da entweder trotz Remissionseintritt eine Therapieintensivierung erfolgte oder aber trotz hoher Krankheitsaktivität eine solche unterblieb. Das Befolgen der Treat-to-Target-Strategie führte im Vergleich zum Nichtbefolgen zu keiner wesentlich höheren DAS44- oder DAS28-ESR-Remissionrate 3 Monate später, erhöhte aber die Chancen auf eine Remission gemäß CDAI-, SDAI- sowie ACR/EULAR-Definition. Eine nachhaltige Treat-to-Target-Strategie (Befolgen der Strategie an mindestens 2 aufeinander folgenden Terminen) ging mit einer signifikant höheren Wahrscheinlichkeit für eine DAS44-Remission (Odds Ratio 1,19; $95 \%$ KI 1,03-1,39) sowie für eine ACR/EULAR-Remission (Odds Ratio 1,49; $95 \%$ KI 1,24-1,81) einher.
FAZIT

Das Treat-to-Target-Konzept funktioniert auch in der Praxis, so das Fazit der Autoren: RA-Patienten profitieren hinsichtlich der Remissionschancen von der Behandlungsstrategie - insbesondere wenn es an mehreren Betreuungsterminen in Folge korrekt angewendet wird. Angesichts dieser Studienergebnisse sollten ihrer Ansicht nach alle Rheumatologen ihren Patienten diese Therapiestrategie anbieten.

Dr. med. Judith Lorenz, Künzell 